\title{
Characterization of shell filling of interacting polarons in a quantum dot through their optical absorption
}

\author{
S. N. Klimin*, V. M. Fomin****, F. Brosens, and J. T. Devreese ${ }^{\sharp, b}$ \\ Theoretische Fysica van de Vaste Stoffen (TFVS), \\ Universiteit Antwerpen (UIA), B-2610 Antwerpen, Belgium
}

(Dated: November 4, 2018)

\begin{abstract}
The method for calculating the ground-state energy and the optical conductivity spectra is developed for a system of a finite number of interacting arbitrary-coupling polarons in a spherical quantum dot with a parabolic confinement potential. The path-integral formalism for identical particles is used in order to take into account the fermion statistics. Using a generalization of the Jensen-Feynman variational principle, the ground-state energy of a confined $N$-polaron system is analyzed as a function of $N$ and of the electron-phonon coupling strength. The calculated optical conductivity spectra of the $N$-polaron system in a quantum dot manifest features related to ground-state transitions between states with different total spin.
\end{abstract}

PACS numbers: 78.20.-e,71.38.+i,71.45.-d,71.45.Gm 


\section{INTRODUCTION}

Many-electron states in quantum dots are intensely investigated by various theoretical methods (see, e. g., Refs. [1, 2, 3, 4] ). These works do not take into account the electronphonon interaction, whereas it can contribute significantly to both the equilibrium and the non-equilibrium properties of quantum dots. In particular, the electron-phonon interaction plays a key role in the optical spectra of some quantum dots (see, e. g., Ref. [5] and references therein). To the best of our knowledge, the many-polaron optical absorption in quantum dots has not yet been widely studied. In Refs. [6, 7], the ground state and the optical response of a fixed number of identical interacting polarons are analyzed using the variational path-integral method for identical particles [8, 9, 10]. As far as we investigate a system with a fixed number of identical particles, it should be described using the canonical ensemble [10]. As distinct from Ref. [6], where only closed-shell systems are considered, the present approach is developed for both closed-shell and open-shell systems.

\section{THEORETICAL APPROACH}

We consider a system of $N$ electrons with Coulomb repulsion, which interact with the lattice vibrations. We assume a spherical quantum dot with a parabolic confinement potential characterized by the frequency $\Omega_{0}$. The partition function of the system is given by a path integral over electron and phonon coordinates (for details, see Ref. [7]). After elimination of the phonon paths, the partition function of the electron-phonon system factorizes into a product of a free-phonon partition function with a partition function $Z_{p}\left(\left\{N_{\sigma}\right\}, \beta\right)$ [where $\left.\beta \equiv 1 /\left(k_{B} T\right)\right]$ of interacting polarons, which is a path integral over the electron coordinates only:

$$
\begin{aligned}
Z_{p}\left(\left\{N_{\sigma}\right\}, \beta\right) & =\sum_{P} \frac{(-1)^{\xi_{P}}}{N_{1 / 2} ! N_{-1 / 2} !} \\
& \times \int d \overline{\mathbf{x}} \int_{\overline{\mathbf{x}}}^{P \overline{\mathbf{x}}} D \overline{\mathbf{x}}(\tau) e^{-S_{p}[\overline{\mathbf{x}}(\tau)]},
\end{aligned}
$$

where $S_{p}[\overline{\mathbf{x}}(\tau)]$ contains the so-called influence phase of the phonons. It describes the phonon-induced retarded interaction between the electrons, including the retarded selfinteraction of each electron with itself. The free energy of a system of interacting polarons $F_{p}\left(\left\{N_{\sigma}\right\}, \beta\right)$ is related to the partition function (11) by the expression

$$
F_{p}\left(\left\{N_{\sigma}\right\}, \beta\right)=-\frac{1}{\beta} \ln Z_{p}\left(\left\{N_{\sigma}\right\}, \beta\right) \text {. }
$$

At present no method is known to calculate the non-gaussian path integral (11) analytically. For distinguishable particles, the Jensen-Feynman variational principle [11] provides a powerful approximation technique. It yields a lower bound to the partition function, and hence an upper bound to the free energy.

It is a non-trivial problem, how a variational principle for the free energy should be formulated for a system of identical particles. However, it was shown [8] that the pathintegral approach to the many-body problem for a fixed number of $N$ identical particles could be formulated in terms of a Feynman-Kac functional on a state space for $N$ indistinguishable 
particles, by imposing an ordering on the configuration space, and the introduction of a set of boundary conditions at the boundaries of this state space. The path integral (with the imaginary-time variable) for identical particles was shown to be positive within this state space. Thus a many-body extension of the Jensen-Feynman inequality was found, which could be used for interacting identical particles (Ref. [8], p. 4476). A more detailed analysis of this variational principle for both local and retarded interactions can be found in Ref. [10]. It is required that the potentials are symmetric with respect to all permutations of the particle positions, and that both the exact propagator and the model propagator are antisymmetric (for fermions) with respect to permutations of any two electrons at any time. This means that both propagators have to be defined on the same configuration space. Under these requirements, the variational inequality for identical particles has the same form as that for the Jensen-Feynman variational principle:

$$
F_{p} \leqslant F_{0}+\frac{1}{\beta}\left\langle S_{p}-S_{0}\right\rangle_{S_{0}},
$$

where $S_{0}$ is a model action with the corresponding free energy $F_{0}$. The angular brackets mean a weighted ave-rage over the paths

$$
\begin{aligned}
\langle(\bullet)\rangle_{S_{0}} & =\frac{1}{Z_{p}\left(\left\{N_{\sigma}\right\}, \beta\right)} \sum_{P} \frac{(-1)^{\xi_{P}}}{N_{1 / 2} ! N_{-1 / 2} !} \\
& \times \int d \overline{\mathbf{x}} \int_{\overline{\mathbf{x}}}^{P \overline{\mathbf{x}}} D \overline{\mathbf{x}}(\tau)(\bullet) e^{-S_{0}[\overline{\mathbf{x}}(\tau)]} .
\end{aligned}
$$

In the present work, we have chosen a model system consisting of $N$ electrons with coordinates $\overline{\mathbf{x}} \equiv\left\{\mathbf{x}_{j, \sigma}\right\}$ and $N_{f}$ fictitious particles with coordinates $\overline{\mathbf{y}} \equiv\left\{\mathbf{y}_{j}\right\}$ in a harmonic confinement potential with elastic interparticle interactions as studied in Ref. [6]. The model confinement frequencies for an electron and for a fictitious particle, the force constants and the mass of a fictitious particle are the variational parameters.

In order to investigate the optical properties of the many-polaron system, we extend in Ref. [7] the memory-function formalism developed in Ref. [12] to the case of interacting polarons in a quantum dot. Within this technique, the optical conductivity for a system of interacting polarons in a parabolic confinement potential is given in terms of the memory function $\chi(\omega)$,

$$
\operatorname{Re} \sigma(\omega)=-\frac{e^{2}}{m} \frac{\omega \operatorname{Im} \chi(\omega)}{\left[\omega^{2}-\Omega_{0}^{2}-\operatorname{Re} \chi(\omega)\right]^{2}+[\operatorname{Im} \chi(\omega)]^{2}},
$$

where $m$ is the electron band mass, and $\chi(\omega)$ is

$$
\begin{aligned}
\chi(\omega) & =\sum_{\mathbf{q}} \frac{2\left|V_{\mathbf{q}}\right|^{2} q^{2}}{3 N \hbar m} \int_{0}^{\infty}\left(e^{i \omega t}-1\right) \\
& \times \operatorname{Im}\left[T_{\omega_{\mathrm{LO}}}^{*}(t)\left\langle\rho_{\mathbf{q}}(t) \rho_{-\mathbf{q}}(0)\right\rangle_{M}\right] d t .
\end{aligned}
$$

Here, $\quad V_{\mathbf{q}}$ is the amplitude of the electron-phonon interaction [12], $T_{\omega}(t)=$ $\cos [\omega(t-i \hbar \beta / 2)] / \sinh (\beta \hbar \omega / 2)$ is the phonon Green's function, and $\left\langle\rho_{\mathbf{q}}(t) \rho_{-\mathbf{q}}(0)\right\rangle_{M}$ is the density-density correlation function calculated using the model Lagrangian of electrons harmonically interacting with fictitious particles. It is noteworthy that the optical conductivity (5) differs from that for a translationally invariant polaron system both by the explicit form of $\chi(\omega)$ and by the presence of the term $\Omega_{0}^{2}$ in the denominator. 


\section{RESULTS AND DISCUSSION}

Further on, we present numerical results for the many-polaron system in a quantum dot at $T=0$. The total spin of interacting polarons in its ground state, as well as the total spin of a confined few-electron system, depends on the number of electrons. The analysis of this dependence reveals a shell structure of quantum dots. In Fig. 11 the total spin $S$ of a finite number of interacting polarons in a spherical quantum dot for different values of the confinement energy $\hbar \Omega_{0}$, of the electron-phonon coupling constant $\alpha$ and of the ratio of the high-frequency and the static dielectric constants $\eta \equiv \varepsilon_{\infty} / \varepsilon_{0}$ is plotted as a function of the number of electrons in a quantum dot. As distinct from few-electron systems without the electron-phonon interaction, three types of spin polarization are possible for the ground state, which can be distinguished from each other using, e. g., capacity measurements.

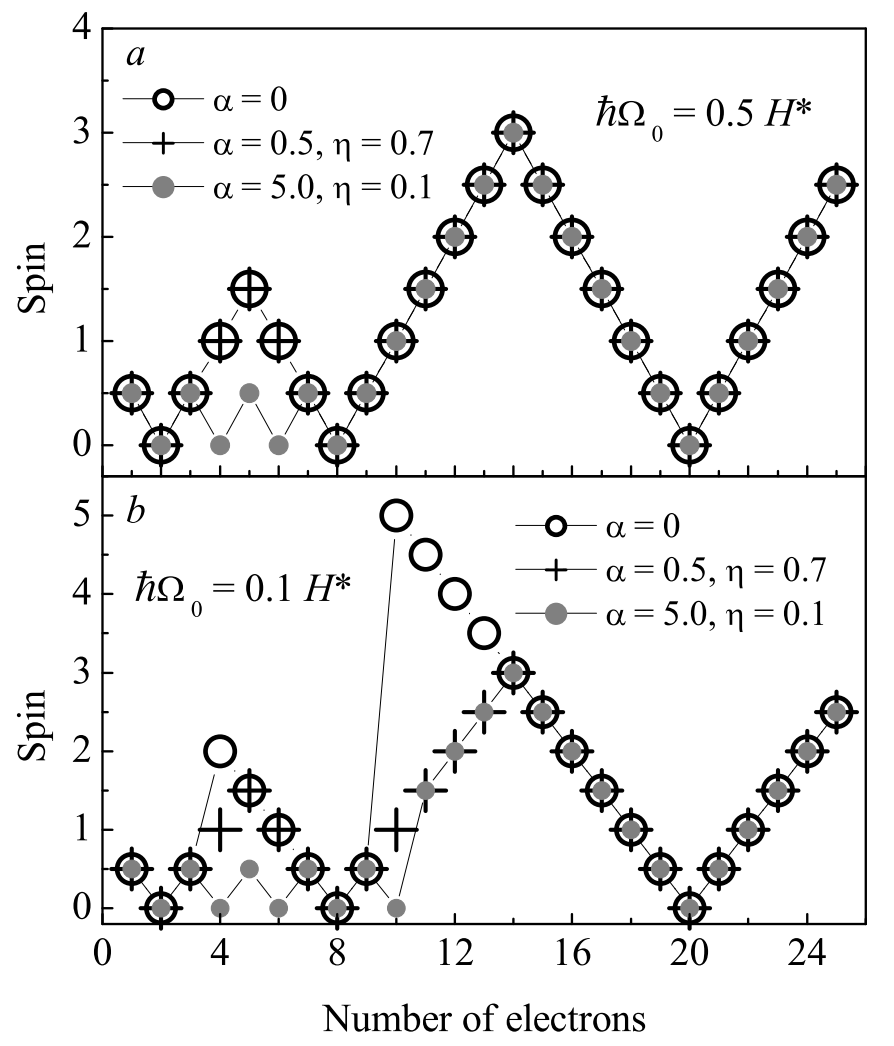

FIG. 1: Total spin of the system of interacting polarons in a quantum dot as a function of the number of electrons for the confinement energy $\hbar \Omega_{0}=0.5 H^{*}(a)$ and for $\hbar \Omega_{0}=0.1 H^{*}(b)$. The confinement energy is measured in effective Hartrees $H^{*}=\left[m /\left(m_{0} \varepsilon_{\infty}^{2}\right)\right]$ Hartree, where $m_{0}$ is the electron mass in the vacuum.

(i) Except the strong-coupling regime and the low-density case, for closed-shell systems $S=0$, while for open-shell systems $S$ takes a maximal value for a given shell filling, in accordance with the Hund's rule. 
(ii) When weakening confinement for a fixed number of electrons, the electron density lowers. Hence, at sufficiently small values of $\Omega_{0}$, a spin-polarized state for a system of interacting polarons in a quantum dot becomes energetically more favorable than a state satisfying the Hund's rule.

(iii) In the strong-coupling regime $(\alpha \gg 1$ and $\eta \ll 1)$, the total spin of an open-shell system for the ground state can take a minimal possible value. This trend to minimize the total spin is a consequence of the electron-phonon interaction, presumably due to the fact that the phonon-mediated electron-electron attraction overcomes the Coulomb repulsion.

The optimal values of the variational parameters are used as input for the calculation of the optical-conductivity spectrum of the system (5). The ground-state transitions between the aforesaid states with different values of the total spin, which occur when changing the number of electrons, are manifested in the optical absorption spectra. In particular, the shell structure for a system of interacting polarons in a quantum dot is clearly pronounced when analyzing the first frequency moment of the optical conductivity,

$$
\langle\omega\rangle=\frac{\int_{0}^{\infty} \omega \operatorname{Re} \sigma(\omega) d \omega}{\int_{0}^{\infty} \operatorname{Re} \sigma(\omega) d \omega} .
$$

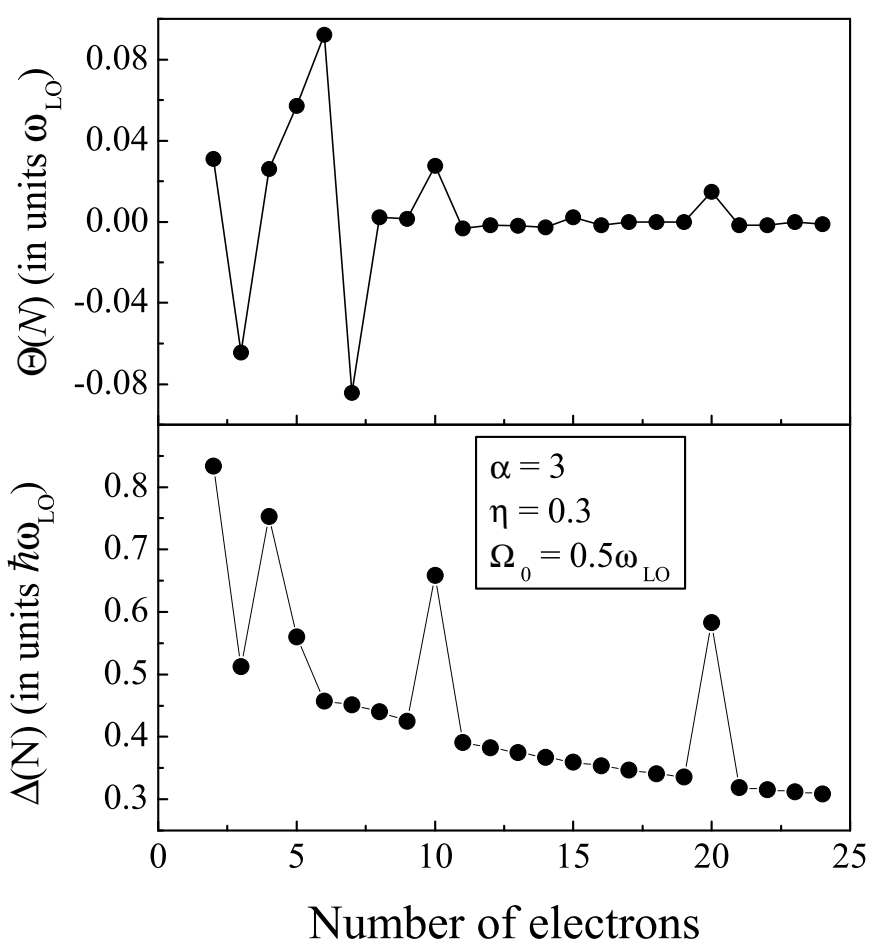

FIG. 2: The function $\Theta(N)(a)$ and the addition energy $\Delta(N)$ (b) for systems of interacting polarons in a quantum dot with $\alpha=3, \eta=0.3$ and $\Omega_{0}=0.5 \omega_{\mathrm{LO}}\left(\hbar \Omega_{0} \approx 0.01361 \mathrm{H}^{*}\right)$.

Figure 2 represents the function

$$
\left.\Theta(N) \equiv\langle\omega\rangle\right|_{N+1}-\left.2\langle\omega\rangle\right|_{N}+\left.\langle\omega\rangle\right|_{N-1}
$$


along with the addition energy $\Delta(N)$ needed to put an extra electron into the quantum dot with $N$ electrons,

$$
\Delta(N)=E^{0}(N+1)-2 E^{0}(N)+E^{0}(N-1),
$$

where $E^{0}(N)$ is the ground-state energy.

Both functions $\Theta(N)$ and $\Delta(N)$ exhibit features at $N=4$ and at $N=7$, where the aforesaid transition occurs. Namely, at $N=4, \Theta(N)$ and $\Delta(N)$ have minima, whereas at $N=7, \Theta(N)$ has a minimum, and $\Delta(N)$ has a kink. Pronounced peaks appear in these functions at the "magic numbers" $N=10$ and $N=20$, which correspond to closedshell systems. The transition between the spin-polarized ground state and the ground state obeying the Hund's rule thus should be observable using optical measurements.

\section{CONCLUSIONS}

The path-integral treatment of the quantum statistics of indistinguishable particles allowed us to find an upper bound to the ground-state energy of a fixed number of polarons in a parabolic confinement potential for arbitrary electron-phonon coupling strength.

The transitions between states with different values of the total spin manifest themselves through discontinuous changes in the optical conductivity spectra and of the addition energy as a function of the number of electrons. Thus, the analysis of the optical-conductivity spectra provides a tool for examining the shell structure of a system of interacting polarons through experimental measurements of the optical absorption in quantum dots.

This work has been supported by the GOA BOF UA 2000, I.U.A.P., F.W.O.-V. projects G.0274.01, G.0435.03, the W.O.G. WO.025.99 (Belgium) and the European Commission GROWTH Programme, NANOMAT project, contract No. G5RD-CT-2001-00545.

[*] Permanent address: Department of Theoretical Physics, State University of Moldova, str. A. Mateevici 60, MD-2009 Kishinev, Republic of Moldova.

[**] Also at: Technische Universiteit Eindhoven, P. B. 513, 5600 MB Eindhoven, The Netherlands.

[ $\sharp]$ Also at: Universiteit Antwerpen (RUCA), Groenenborgerlaan 171, B-2020 Antwerpen, Belgium and Technische Universiteit Eindhoven, P. B. 513, 5600 MB Eindhoven, The Netherlands.

[b] Corresponding author. E-mail: devreese@uia.ua.ac.be

[1] B. Szafran, J. Adamowski, and S. Bednarek, Phys. Rev. B 61, 1971 (2000).

[2] B. Partoens and F. M. Peeters, Phys. Rev. Lett. 84, 4433 (2000).

[3] R. Egger, W. Häusler, C. H. Mak, and H. Grabert, Phys. Rev. Lett. 82, 3320 (1999).

[4] S. M. Reimann, M. Koskinen, and M. Manninen, Phys. Rev. B 62, 8108 (2000).

[5] V. M. Fomin, V. N. Gladilin, J. T. Devreese, E. P. Pokatilov, S.N. Balaban, and S.N. Klimin, Phys. Rev. B 57, 2415 (1998).

[6] J. T. Devreese, S. N. Klimin, V. M. Fomin, and F. Brosens, Solid State Commun. 114, 305 (2000).

[7] S. N. Klimin, V. M. Fomin, F. Brosens, and J. T. Devreese, to be published.

[8] L. F. Lemmens, F. Brosens, and J. T. Devreese, Phys. Rev. E 53, 4467 (1996).

[9] F. Brosens, J. T. Devreese, and L. F. Lemmens, Phys. Rev. E 55, 227 (1997); 55, 6795 (1997); 58, 1634 (1998). 
[10] J. T. Devreese, in: Fluctuating Paths and Fields (World Scientific, Singapore, 2001), pp. 289-304.

[11] R. P. Feynman, Statistical Mechanics (Benjamin, Massachusetts, 1972).

[12] J. Devreese, J. De Sitter, and M. Goovaerts, Phys. Rev. B 5, 2367 (1972). 